\title{
Segurança Alimentar e Nutricional e fatores associados em municípios baianos de diferentes portes populacionais
}

\author{
Marlus Henrique Queiroz Pereira1, Maria Luiza Amorim Sena Pereira², Barbara \\ Eduarda Panelli-Martins ${ }^{3}$ e Sandra Maria Chaves dos Santos ${ }^{4}$
}

O artigo apresenta resultados da avaliação da Insegurança Alimentar (IA) em municípios da Bahia, Brasil. Aplicou-se um protocolo com 23 indicadores que medem a Segurança Alimentar e Nutricional nas quatro dimensões (disponibilidade de alimentos, acesso aos alimentos, consumo dos alimentos e utilização biológica dos nutrientes). Foram investigados 57 municípios de diferentes portes (pequeno, médio e grande). Foram utilizados estatísticas descritivas e os testes de Correlação de Pearson e Qui-quadrado de Pearson. Todos os municípios foram classificados em situação de IA (24,6\% IA leve, $71,9 \%$ IA moderada e 3,5\% IA grave) e houve uma correlação $(r=0,369)$ estatisticamente significante $(p<0,01)$ entre SAN/IA e o número de habitantes dos municípios. A dimensão acesso se mostrou estatisticamente significativa com os indicadores: baixa renda, número de moradores no domicílio, baixa escolaridade e com a renda média domiciliar per capita $(p<0,01)$.

Palavras-chave: Segurança Alimentar e Nutricional, fatores associados, indicadores.

\section{Food and Nutrition Security and Associated Factors in Bahia Cities with Different Population Sizes}

This article provides the results of a Food Insecurity (FI) assessment conducted in cities from Bahia, Brazil. A protocol with 23 indicators was applied that measures four dimensions of Food and Nutrition Security (availability of food; food consumption; access to food; and biological use of nutrients). We investigated 57 different-sized cities (small, medium and large). Descriptive statistics and Pearson's correlation and Pearson's Chi-square tests were used. All cities were in a condition of FI $(24.6 \%$ with mild FI, $71.9 \%$ posting a moderate FI and 3.5\% severe FI) and there was a statistically significant correlation $(r=0.369)(p<0.01)$ between FS/FI and the number of inhabitants of cities. The access dimension was statistically significant with the indicators: ratio of people with low income; number of residents per household; population with less than 4 years of study; and average per capita monthly household income $(p<0.01)$.

Keywords: Food and Nutrition Security, associated factors, indicators.

1 Professor Assistente da Universidade Federal do Oeste da Bahia (UFOB) - Centro das Ciências Biológicas e da Saúde. Endereço para correspondência: Rua Professor José Seabra de Lemos no 316, Barreiras, Bahia, Brasil. CEP: 47808-021. Telefone: (77) 3614-3241. Fax: (77) 3614-3500. E-maik. marlus.pereira@ufob.edu.br

2 Professora Assistente da Universidade Federal do Oeste da Bahia (UFOB) - Centro das Ciências Biológicas e da Saúde. Barreiras, Bahia, Brasil.

${ }^{3}$ Professora da Universidade Federal do Recôncavo da Bahia (UFRB) - Centro de Ciências da Saúde. Santo Antônio de Jesus, Bahia, Brasil.

${ }_{4}^{4}$ Professora Associada da Universidade Federal da Bahia (UFBA) - Escola de Nutrição. Pós-Graduação em Alimentos, Nutrição e Saúde. Salvador, Bahia, Brasil. 


\section{INTRODUÇÃO E OBJETIVOS}

Nos últimos anos no Brasil, dados das Pesquisas Nacional por Amostragem de Domicílio (PNAD) realizadas pelo Instituto Brasileiro de Geografia e Estatística (IBGE), mostram melhora nos resultados da Segurança Alimentar e Nutricional (SAN) da população, com redução da situação de Insegurança Alimentar (IA). Tal condição tem sido atribuída ao desenvolvimento de diversos programas e políticas públicas voltados à promoção da SAN para população brasileira, a exemplo do Programa Bolsa Família e do Programa de Saúde da Família. Entretanto, mesmo diante dessa conjuntura 14,7 milhões de domicílios particulares convivem ainda com situações de privação e fomel ${ }^{[1,2,3]}$.

A SAN é um fenômeno multidimensional, contemplando pelo menos quatro dimensões: a) disponibilidade de alimentos; b) consumo de alimentos; c) acesso aos alimentos; d) utilização biológica de nutrientes ${ }^{[4,5]}$. A Escala Brasileira de Insegurança Alimentar (EBIA), instrumento utilizado para avaliar a SAN, mede determinados aspectos, predominantemente relacionados à dimensão do acesso aos alimentos, a partir de dados primários coletados em domicílios particulares. Assim, para orientar políticas públicas, faz-se necessário contar com avaliações que possam contemplar outras dimensões do problema, em diferentes contextos ${ }^{[6,7,8,9]}$. Nesse sentido, pode-se notar ainda que são escassos os estudos multidimensionais de avaliação da SAN em âmbito municipal, principalmente pesquisas que envolvam mais de um município[10]. Entre os fatores que justificam esse cenário, destaca-se a dificuldade de acesso aos dados ou até mesmo inexistência dos mesmos nos municípios, bem como a falta de métodos viáveis em termos técnicos e operacionais que possibilitem essas investigações.

Alguns pesquisadores e instituições, como a Food and Agriculture of the United Nations (FAO) apontam para a relevância da criação de protocolos, com múltiplos indicadores, que consigam avaliar a SAN nas suas diferentes vertentes, correspondendo de forma mais adequada às realidades locais analisadas $[9,11,12]$.
Nesse sentido, foi desenvolvido e testado no período entre 2004 a 2008, um protocolo de indicadores para avaliação da SAN em âmbito municipal no Brasil, o qual mostrou ser uma ferramenta de fácil utilização, baixo custo, e capaz de refletir as situações de SAN, e IA em três níveis: leve, moderada e grave[13,14]. Devido a recentes mudanças na forma de implementação das políticas sociais de promoção da SAN no Brasil, foi elaborada uma proposta de atualização e aperfeiçoamento desse instrumento. O protocolo utiliza dados secundários disponíveis em base de dados públicas, e avalia a SAN nas quatro dimensões, sendo possível reconhecer em cada dimensão, os fatores que facilitam ou dificultam a condição de SAN[13,15].

No Brasil, municípios de médio e pequeno porte dispõem de poucos resultados oriundos da avaliação da SAN, cuja viabilidade para a realização de pesquisas encontra-se prejudicada. Frente a isso, faz-se imperativa aplicação de metodologias que permitam a geração de dados sobre a SAN capazes de subsidiar a implementação de políticas públicas nessa direção e permitam a sua replicação. Assim, o presente estudo tem como objetivo avaliar situação de SAN/IA e fatores associados, em um conjunto de municípios no estado da Bahia, por meio da aplicação de uma nova abordagem metodológica, o protocolo multidimensional de indicadores.

\section{MATERIAL E MÉTODOS}

\section{Cenário do Estudo e Amostra}

O estudo foi realizado em uma amostra intencional de municípios de diferentes portes (pequeno, médio e grande) nos 27 Territórios de Identidade (TI) do Estado da Bahia, Brasil.

Segundo o Instituto Brasileiro de Geografia e Estatística (IBGE), os municípios de pequeno porte são aqueles com menos de 50 mil habitantes; médio porte, entre 50 a 100 mil; e grande porte, aqueles acima de 100 mil habitantes. Essa classificação, conforme o porte do município em cada território de identidade, foi escolhida para possibilitar a aplicação do protocolo em diferentes cenários municipais, que em cada caso, assume uma configuração, sob influência de fatores políticos, 
geográficos, administrativos, econômicos, sociais e culturais ${ }^{[16]}$.

O Território de Identidade é conceituado como um espaço físico, geograficamente definido, geralmente contínuo, caracterizado por critérios multidimensionais, tais como o ambiente, a economia, a sociedade, a cultura, a política e as instituições, e uma população com grupos sociais relativamente distintos, que se relacionam interna e externamente por meio de processos específicos, onde se pode distinguir um ou mais elementos que indicam identidade, coesão social, cultural e territorial[17].

Em cada um dos 27 TI propôs-se a seleção aleatória de 3 municípios, sendo um de pequeno porte, um de médio e um de grande porte. No entanto, em 7 TI não foram identificados municípios de médio porte e em 17 TI não houve municípios de grande porte. Assim em alguns territórios foram selecionados um município, em outros dois, ou três. Desta forma, foram avaliados 57 municípios, assim distribuídos: 27 de pequeno porte, 20 de médio, e 10 de grande porte.

\section{Protocolo de Indicadores}

O protocolo utilizado para a avaliação da SAN nos municípios é composto por 23 indicadores, agrupados nas quatro dimensões da avaliação da SAN (DA: Disponibilidade de Alimentos; AA: Acesso aos Alimentos; CA: Consumo de Alimentos; UBN: Utilização Biológica dos Nutrientes). Os resultados obtidos foram classificados de forma dicotômica (adequado ou inadequado) ou politômica (adequado, aceitável ou inadequado) [15,18]. Os indicadores contemplados no protocolo avaliam a SAN entendendo a sua complexidade e interdisciplinaridade, desta forma, perpassam por vários aspectos relevantes como saúde, estado nutricional, educação, renda, emprego, clima, saneamento, agricultura familiar, entre outros (Quadro 1).

Os indicadores escolhidos para compor o protocolo foram aqueles que melhor se adequaram às propriedades (relevância social, confiabilidade e a validade de constructo), e características de sensibilidade, especificidade, inteligibilidade, periodicidade, factibilidade, historicidade desejáveis para avaliação da situação de $S A N[13,19,20]$.

\section{Coleta de Dados}

Todos os indicadores utilizados no protocolo foram construídos a partir de dados secundários, oriundos de pesquisas oficiais do governo, obtidos nas principais bases brasileiras de acesso público online: o Instituto Brasileiro de Geografia e Estatística, o Departamento de Informática do Sistema Único de Saúde (DATASUS), bem como nos relatórios sociais da Secretaria de Avaliação e Gestão da Informação (SAGI) do então Ministério de Desenvolvimento Social e Combate à Fome (MDS). As fontes, juntamente com outras informações sobre as premissas, fórmulas, critérios, parâmetros e pontuações dos indicadores foram utilizadas segundo o detalhamento proposto por Pereira ${ }^{[15]}$. Todos os dados coletados foram armazenados em uma versão do protocolo utilizando o software Microsoft Office Excel versão 2007. A coleta foi realizada no período de agosto a outubro de 2013.

\section{Análise e Processamento dos Dados}

Para análise dos dados, foi elaborada uma matriz contendo uma fórmula ou o critério de obtenção de cada indicador, seguido de parâmetros para classificação, pontuação, meios de verificação e premissas de cada indicador ${ }^{[15]}$.

Foi atribuída então, para cada indicador uma pontuação de 0 a 10 , sendo o valor zero associado com resultados indicativos de risco de IA e o valor 10 associado com resultados positivos para a SAN. Desta forma, em valores absolutos, o protocolo apresentou uma escala de pontuação de 0 a 230 pontos no total. Para análise criou-se uma escala de pontuação da SAN, relativa ao percentual obtido em quartis, fazendo corresponder a cada intervalo uma situação de $\mathrm{SAN}$, Insegurança Alimentar leve, moderada ou grave (Quadro 2). 
Quadro 1. Composição do protocolo de indicadores por dimensão da Segurança Alimentar e Nutricional

Dimensão: Disponibilidade de Alimentos

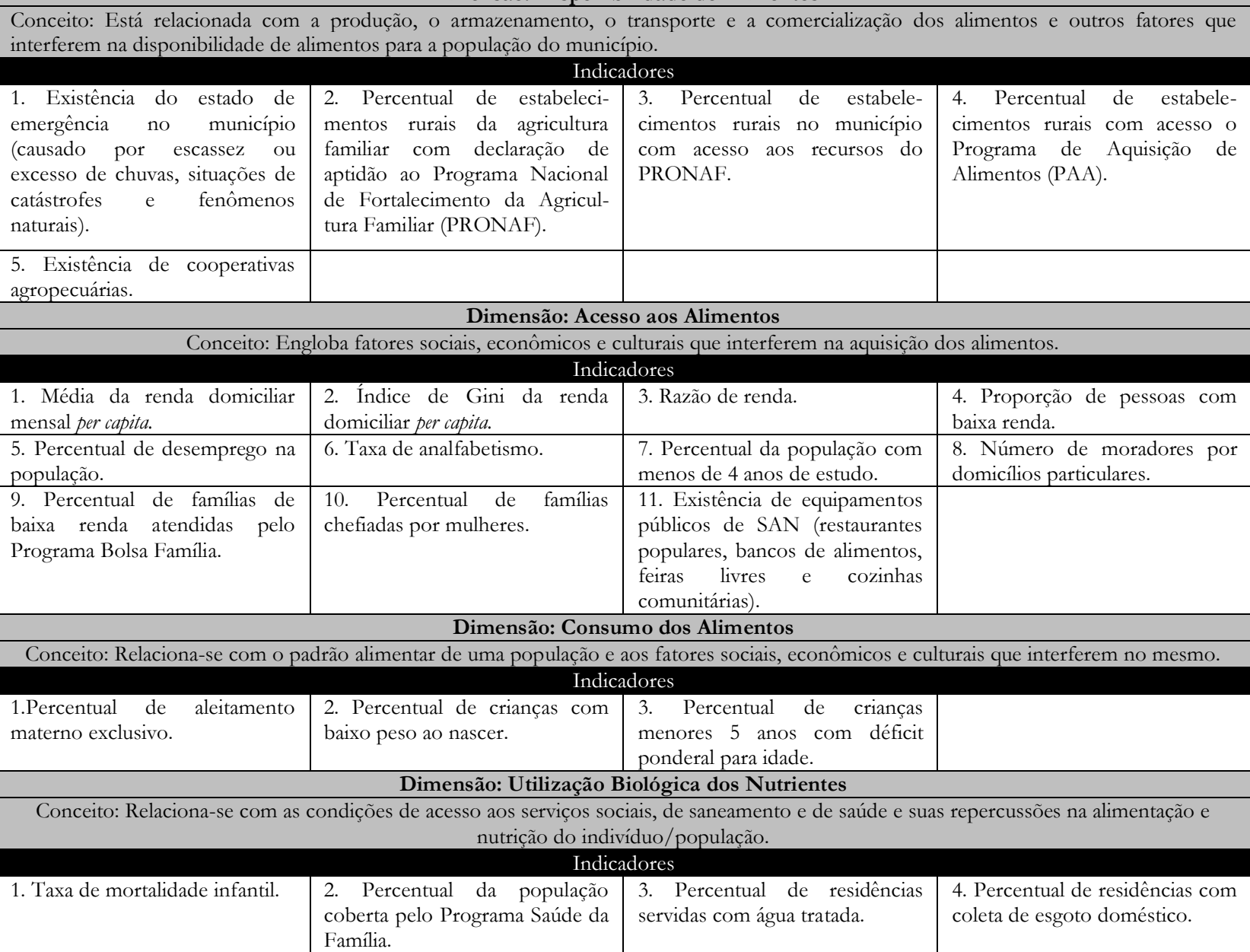

Quadro 2. Escala de pontuação (percentuais) e categorias para avaliação da SAN municipal com uso de um protocolo de indicadores

\begin{tabular}{|c|c|c|c|}
\hline IAG & IAM & IAL & SAN \\
\hline 0 a $24,9 \%$ & 25 a $49,9 \%$ & 50 a $74,9 \%$ & 75 a $100 \%$ \\
\hline $\begin{array}{l}\text { Resultados dos indicadores } \\
\text { sinalizam para existência de } \\
\text { muitos fatores comprometedores } \\
\text { da SAN, implicando em } \\
\text { situações de privação de } \\
\text { alimentos e fome }\end{array}$ & $\begin{array}{l}\text { Resultados dos indicadores } \\
\text { sinalizam para existência de } \\
\text { alguns fatores comprometedores } \\
\text { da SAN, com risco de situações } \\
\text { de privação de alimentos e fome. }\end{array}$ & $\begin{array}{l}\text { Resultados dos indicadores } \\
\text { sinalizam para existência de } \\
\text { alguns fatores comprometedores } \\
\text { da SAN, sem caracterizar risco } \\
\text { de privação de alimentos e fome. }\end{array}$ & $\begin{array}{l}\text { Resultados } \text { dos } \\
\text { sinalizam indicadores } \\
\text { favoráveis à promoção da SAN } \\
\text { no município. }\end{array}$ \\
\hline
\end{tabular}


As análises estatísticas foram realizadas utilizando o software Statistical Packadge Social of Sciences (SPSS), versão 16.0. Foi realizada análise descritiva para medir a frequência das variáveis de estudo e em seguida, buscando verificar a correlação entre a situação de SAN/IA (escore total do protocolo) e o tamanho do município (número de habitantes), bem como cada um dos indicadores utilizados, foi aplicado o Teste de Correlação de Pearson para variáveis quantitativas, considerando nível de significância $<5 \%$ e o teste Qui-quadrado de Pearson para variáveis qualitativas.

\section{RESULTADOS}

Considerando os resultados globais do protocolo, todos os municípios foram classificados em situação de IA, sendo $24,6 \%$ em IA leve, $71,9 \%$ em IA moderada e 3,5\% em IA grave. Entre os municípios de pequeno e médio porte predominou a IA moderada $(74,1 \%$ e $94,7 \%$, respectivamente), enquanto nos municípios de grande porte observou-se predominância de IA leve $(72,7 \%)$. Observou-se IA grave somente nos municípios de pequeno porte $(7,4 \%)$ (Figura 1). Houve correlação $(r=0,369)$ entre o escore total do protocolo e o número de habitantes, sendo estatisticamente significante $p=0,005)$. Ou seja, quanto maior o porte do município, melhores serão as condições de SAN.

Figura 1. Distribuição da prevalência de Insegurança Alimentar, segundo porte populacional, em 57 municípios do Estado da Bahia, Brasil, com a utilização do protocolo de indicadores.

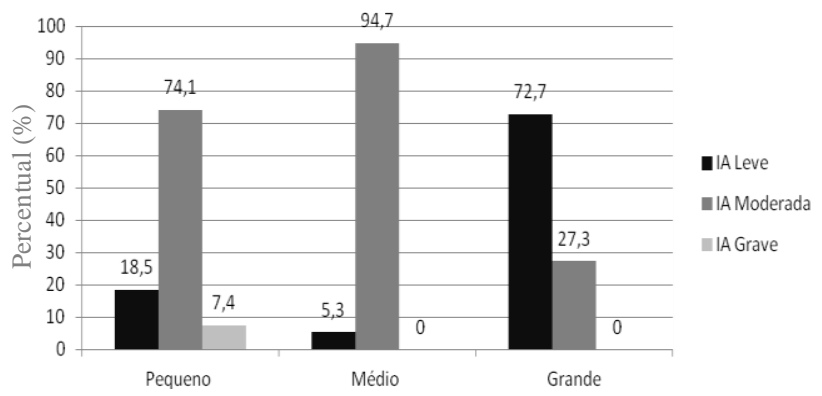

Os resultados da avaliação por dimensão revelaram que as condições favoráveis ou constrangedoras ao alcance da SAN diferem segundo as categorias (Tabela 1). $\mathrm{Na}$ disponibilidade de alimentos 93\% dos municípios foram classificados em situação de IA, e somente 7\% dos municípios foram identificados em condições favoráveis para alcance da SAN. No mesmo sentido, no acesso aos alimentos todos os municípios foram classificados em situação de IA, com destaque para um total de $87,7 \%$ em IA moderada e grave. Também na dimensão de consumo de alimentos, a maioria (52,6\%) dos municípios foi classificada na condição de IA moderada e grave. Por outro lado, a utilização biológica de nutrientes foi aquela que apresentou o maior percentual $(22,8 \%)$ de municípios em condições favoráveis à situação de SAN, embora grande parte $(77,2 \%)$ dos municípios apresentasse algum grau de IA.

$\mathrm{Na}$ análise isolada por indicador, considerando os parâmetros adequado, aceitável e inadequado, foi possível observar quais os aspectos foram mais relacionados a SAN: razão de renda $(98,2 \%$ dos municípios obtiveram classificação adequada para este indicador), taxa de desemprego (96,5\% dos municípios), percentual de crianças com déficit ponderal $(96,5 \%)$, percentual de famílias atendidas pelo Programa Bolsa Familia (93\%), taxa de mortalidade infantil $(82,5 \%)$ e percentual da população coberta pela Estratégia de Saúde da Família (82,5\%). Entretanto, para os outros indicadores, os municípios apresentaram uma baixa classificação para o adequado, sendo considerados fatores relacionadas a IA: o percentual de familias chefiadas por mulheres $(7 \%)$, percentual de estabelecimentos rurais com acesso ao Programa de Aquisição de Alimentos (7\%), existência de equipamentos públicos de SAN (10,5\%) e número de moradores por domicílio particular (19,3\%) (Figura 2).

Quanto à correlação entre o escore total do protocolo (condição de SAN) e cada indicador utilizado, observou-se que os indicadores percentual de estabelecimentos rurais com acesso ao PRONAF, média da renda domiciliar per capita, percentual de residências com coleta de esgoto doméstico, percentual de residências com abastecimento de água, percentual de estabelecimentos rurais com declaração de aptidão ao PRONAF e existência de cooperativas agropecuárias apresentaram correlação positiva estatisticamente significante. Enquanto os indicadores, percentual de famílias de baixa renda atendidas com o Programa Bolsa Família, taxa de analfabetismo, percentual da população com menos de quatro anos de estudo, número de moradores por domicílio e proporção da população de baixa renda apresentaram correlação negativa estatisticamente significante. Os 
demais indicadores não apresentaram correlação estatisticamente significante na amostra estudada, embora possam ter relevância em outros contextos (Tabela 2). Para os indicadores, municípios em situação de emergência e existência de equipamentos públicos de SAN, foi aplicado o teste qui-quadrado de Pearson e em ambos não foi observada significância estatística na associação com a variável desfecho.

\section{DISCUSSÃO}

Neste estudo, com a aplicação do protocolo de indicadores, a insegurança alimentar foi uma condição presente em todos os municípios avaliados. Foram observadas em municípios de pequeno, médio e grande porte desde condições mais leves, em que poucos fatores podem comprometer o estado de SAN, até situações mais graves, em que diversos aspectos apontam para uma condição de privação de alimentos, nutrientes e fome.

Resultados semelhantes de IA, principalmente moderada e grave, porém em uma perspectiva domiciliar, foram encontrados no Uruguai, Guatemala e Colômbia, países latino-americanos que compartilham características sociais, econômicos, culturais e de saúde com o Brasil[21,22,23]. O último relatório mundial da FAO, publicado em 2015, estimou que 10,9\% (794,6 milhões de pessoas) da população mundial apresentava desnutrição, um dos graus da Insegurança Alimentar. Desse total, 34,3 milhões de pessoas estão na América Latina e no Caribe[24].

Tabela 1. Prevalência de SAN e IA em 57 municípios do Estado da Bahia, Brasil, segundo as dimensões de avaliação da SAN

\begin{tabular}{ccccc}
\hline Situação de SAN/IA & $\begin{array}{c}\text { Disponibilidade } \\
\mathbf{n ~ ( \% )}\end{array}$ & $\begin{array}{c}\text { Acesso } \\
\mathbf{n ~ ( \% )}\end{array}$ & $\begin{array}{c}\text { Consumo } \\
\mathbf{n}(\mathbf{\%})\end{array}$ & $\begin{array}{c}\text { Utilização } \\
\mathbf{n}(\mathbf{\%})\end{array}$ \\
\hline Segurança Alimentar e Nutricional & $4(7,0)$ & $0(0)$ & $3(5,3)$ & $13(22,8)$ \\
Insegurança Alimentar Leve & $16(28,1)$ & $7(12,3)$ & $24(42,1)$ & $20(35,1)$ \\
Insegurança Alimentar Moderada & $20(35,1)$ & $43(75,4)$ & $17(29,8)$ & $22(38,6)$ \\
Insegurança Alimentar Grave & $17(29,8)$ & $7(12,3)$ & $13(22,8)$ & $2(3,5)$ \\
Total & $57(100)$ & $57(100)$ & $57(100)$ & $57(100)$ \\
\hline
\end{tabular}

Figura 2. Prevalência de adequação de cada indicador do protocolo entre os 57 municípios avaliados

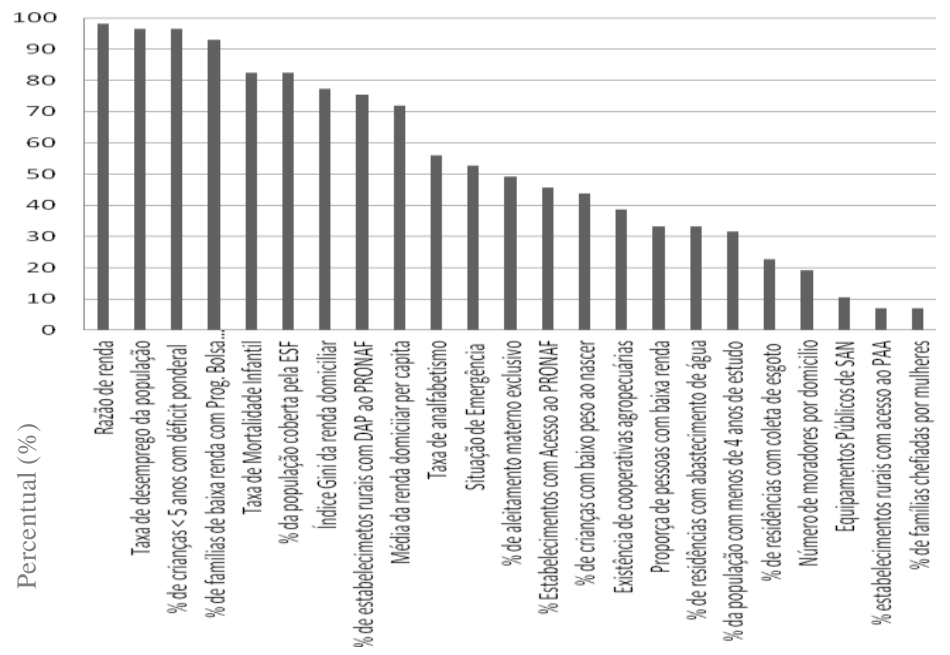


No Brasil, segundo a Pesquisa Nacional por Amostra de Domićlios (PNAD) divulgada em 2013, a IA afeta $22,6 \%$ dos domićlios particulares pesquisados, com destaque para os $7,8 \%$ de IA moderada e grave. Especificamente na região nordeste do Brasil, uma das regiões mais vulneráveis econômica e socialmente, e onde foi também realizado o presente estudo, os resultados revelaram que $38,1 \%$ dos domicilios foram classificados em Insegurança Alimentar, sendo que $14,5 \%$ estavam em IA moderada e grave ${ }^{[1}$. Além da pesquisa nacional sobre IA junto às PNADs, estudos localizados adotando a EBIA vêm sendo realizados e revelando situações diversas de Insegurança Alimentar[14, 25, 26].

$\mathrm{Na}$ avaliação da SAN por dimensão percebese que as dimensões de disponibilidade, acesso e consumo de alimentos apresentaram uma elevada prevalência de IA moderada e grave. Nestas dimensões foram avaliados aspectos como condições climáticas; produção, organização e financiamento agropecuário; fatores econômicos e sociais; e indicadores antropométricos. Resultados que dialogam com esse panorama estão presentes no estudo de Gubert et al. [10] e Hoffman ${ }^{[27]}$, os quais avaliaram a insegurança alimentar domiciliar por meio de pesquisas populacionais realizadas no Brasil. Ambos os trabalhos apontam desafios a serem superados nessas dimensões: modelos de produção, questões ambientais, escolaridade, baixa renda, desemprego e efetivação dos programas sociais.

A análise que mostrou quais indicadores estavam mais adequados nos municípios, revelou que os indicadores relacionados à concentração de renda, desemprego, programas sociais e de saúde, apresentaram os melhores resultados, apontando talvez, melhorias nas condições de vidas nos municípios. Possivelmente, esse cenário reflete o conjunto de políticas sociais e de saúde que foram implementadas desde a década de 90, sendo intensificadas a partir dos anos 2000, com destaque para o Programa de Transferência de Renda (Bolsa Família), valorização do salário mínimo, estimulo ao emprego, bem como a Estratégia de Saúde da Família[24]. No estudo de Aliaga e Chaves-dosSantos ${ }^{288}$, ao analisar a relação entre as ações públicas de enfrentamento da IA e as variáveis de desenvolvimento (econômicas, sociais entre outras) entre os países participantes da Cúpula Mundial da Alimentação (CMA) em 1996, observou-se que a maioria dos países signatários implantaram essas ações, principalmente os menos desenvolvidos. Essas ações foram desenvolvidas diante de um cenário influenciado pela vulnerabilidade da população, por cooperações internacionais e pelo interesse político nesses países.

Dos seis indicadores que apresentaram correlação positiva com o escore total do protocolo, que demonstra o estado de SAN/IA, três deles estavam na dimensão de disponibilidade de alimentos, a saber: percentual de estabelecimentos rurais com acesso ao PRONAF, Existência de cooperativas agropecuárias e percentual de estabelecimentos rurais com DAP ao PRONAF. Esses indicadores apontam para a estreita relação e interdependência entre a agricultura familiar e a situação de SAN nos municípios. Ou seja, quanto mais favoráveis às condições para esse modo de produção de alimentos, como uma rede consolidada de produção e comercialização para a agricultura familiar, mais chances de o município estar em uma condição de SAN. Os estudos realizados no Brasil por Santos et al[29] e Silva ${ }^{[30]}$ que analisaram a relação da SAN com a agricultura familiar, sugerem que iniciativas como o Programa de Aquisição de Alimentos e formação de cooperativas, além de favorecerem a produção e diversificação dos alimentos, garantem também uma ampliação da renda e da qualidade de vida dos agricultores, possibilitando assim, um cenário mais adequado para o alcance da SAN. Nessa mesma direção, Baiphethi e Jacobss ${ }^{[1]}$ em um trabalho realizado com agricultores familiares da África do Sul relata que os alimentos produzidos por esse tipo de agricultor podem desempenhar um papel importante na redução da vulnerabilidade das famílias rurais e urbanas em situações de Insegurança Alimentar, melhorando os meios de subsistência e ajudando a aliviar a alta inflação dos preços dos alimentos.

Os indicadores relacionados ao acesso aos serviços essenciais, como água e esgotamento sanitário, também apresentaram uma correlação positiva com a variável desfecho. Desta forma, os municípios que apresentaram as maiores coberturas da rede de abastecimento de água e esgotamento sanitário, foram aqueles que tiveram as condições mais favoráveis para o estado de SAN. Resultados parecidos foram encontrados no estudo de Monego et al.[32] ao estudar comunidades quilombolas na região norte do Brasil, indicando que variáveis como, tipo de 
abastecimento de água e esgotamento sanitário tiveram associação estatisticamente significante com a SAN. Rede de abastecimento de água e esgotamento sanitário insatisfatória ou insuficiente pode comprometer a aproveitamento dos alimentos e dos nutrientes pelo organismo dos indivíduos, tanto pela contaminação microbiológica, quanto física, bem como torna indivíduos e populações mais vulneráveis a patologias infecciosas e parasitárias que impactarão na saúde de forma geral e no estado nutricional[33].

Tabela 2. Coeficiente de correlação entre Segurança/Insegurança Alimentar (escore total do protocolo) e indicadores utilizados no protocolo de indicadores

\begin{tabular}{|c|c|c|}
\hline Indicador & $\boldsymbol{r}$ & $p$-valor \\
\hline \multicolumn{3}{|c|}{ Dimensão de Disponibilidade de Alimentos } \\
\hline Percentual de estabelecimentos rurais com Declaração de Aptidão ao PRONAF & 0,337 & 0,010 \\
\hline Percentual de estabelecimentos rurais com acesso ao PRONAF & 0,480 & 0,000 \\
\hline Estabelecimentos rurais com acesso ao Programa de Aquisição de Alimentos (PAA) & 0,180 & 0,180 \\
\hline Existência de cooperativas agropecuárias & 0,331 & 0,012 \\
\hline \multicolumn{3}{|l|}{ Dimensão de Acesso aos Alimentos } \\
\hline Média da Renda domiciliar per capita & 0,397 & 0,002 \\
\hline Índice Gini da renda domiciliar & 0,142 & 0,292 \\
\hline Razão de renda & $-0,251$ & 0,060 \\
\hline Proporção de pessoas com baixa renda & $-0,508$ & 0,000 \\
\hline Percentual de desemprego da população & 0,011 & 0,936 \\
\hline Taxa de analfabetismo & $-0,465$ & 0,000 \\
\hline Percentual da população com menos de 4 anos de estudo & $-0,475$ & 0,000 \\
\hline Número de moradores por domicílio particular & $-0,486$ & 0,000 \\
\hline Famílias atendidas pelo Programa Bolsa Família & $-0,266$ & 0,046 \\
\hline Famílias chefiadas por mulheres & 0,226 & 0,092 \\
\hline \multicolumn{3}{|c|}{ Dimensão de Consumo dos Alimentos } \\
\hline Aleitamento materno exclusivo & 0,232 & 0,083 \\
\hline Crianças com baixo peso ao nascer & $-0,143$ & 0,288 \\
\hline Crianças menores de 5 anos com déficit ponderal para idade & $-0,197$ & 0,145 \\
\hline \multicolumn{3}{|c|}{ Dimensão de Utilização Biológica dos Nutrientes } \\
\hline Taxa de mortalidade infantil & 0,164 & 0,231 \\
\hline População coberta pela Estratégia de Saúde da Família & 0,055 & 0,686 \\
\hline Residências com abastecimento de água & 0,355 & 0,007 \\
\hline Residências com coleta de esgoto doméstico & 0,395 & 0,002 \\
\hline
\end{tabular}

$\mathrm{O}$ indicador renda média per capita também apresentou uma correlação positiva com a SAN nos municípios avaliados. Sendo assim, quanto maior a renda média dos domicílios, maiores serão as condições para o alcance da SAN. Estudos realizado no Reino Unido, Nigéria e Brasil, também apontam para essa relação entra renda familiar e SAN[34,35,36]. A melhoria na renda, possivelmente está relacionada com a melhoria nos níveis de escolaridade e emprego dos munícipes, bem como a focalização dos programas sociais de enfretamento da pobreza e miséria e o suporte social, aspectos que favorecem a situação de SAN $[27,37$.
Todos os indicadores que se correlacionaram negativamente com a situação de SAN pertenciam à dimensão de acesso aos alimentos: taxa de analfabetismo, percentual da população com menos de 4 anos de estudo, percentual da população com baixa renda, percentual de famílias de baixa renda atendida pelo Programa Bolsa Família e número de moradores por domicílio particular. Ou seja, quanto maior os resultados encontrados para esses indicadores, mais desfavoráveis as condições para o estado de SAN do município. Resultados semelhantes foram encontrados no estudo de Calmom et al.[18], que também utilizou o mesmo protocolo de indicadores aplicado no presente estudo para avaliar a situação de SAN em 414 municípios em um estado do nordeste brasileiro, 
porém abordando somente a dimensão de Acesso aos Alimentos. O referido estudo também mostrou que os indicadores de baixa renda e o percentual da população com menos de 4 anos de estudos foram os aspectos que mais contribuíram para o estado de IA. Hoffman ${ }^{[27]}$ e Bezerra et al. [38], da mesma forma, demonstraram um cenário de determinação social da Insegurança Alimentar, ocasionado principalmente pela baixa renda, pouca escolaridade e elevada quantidade de indivíduos no domicilio.

Vale mencionar que o estudo também apresenta algumas limitações, principalmente referente a utilização de dados secundários, como: exclusão de alguns indicadores relevantes para o tema, diante da inexistência de dados municipais; bem como pela instabilidade das bases de dados públicas, diante dos cenários políticos do país. Mesmo assim, o protocolo utilizado neste estudo mostra-se como mais uma ferramenta para a compreensão da SAN no cenário nacional.

\section{CONCLUSÃO}

A insegurança alimentar foi uma condição presente de maneira expressiva no resultado deste estudo, estando presente em todos os municípios avaliados, com predomínio para IA moderada e grave, sendo evidenciada em municípios de pequeno e médio porte. Essa situação de IA foi identificada em todas as dimensões do fenômeno, mas sob uma perspectiva estatística, houve correlação significante principalmente entre indicadores da dimensão de acesso aos alimentos e a referida condição, com destaque para os fatores renda, escolaridade e número de moradores por domicílio.

Os resultados de SAN e IA obtidos pela aplicação do protocolo são compatíveis com resultados encontrados com a utilização de outras metodologias de avaliação em outros estudos, com a vantagem de ser possível identificar que condições estão gerando as situações observadas. Diante dessas observações, ressalta-se ainda a necessidade de atualizações frequentes de protocolos de avaliação da SAN/IA, no sentido de acompanhar mudanças políticas, sociais e econômicas, que permite a melhor interpretação dessa condição nos municípios.

\section{AGRADECIMENTOS}

À Fundação de Amparo à Pesquisa do Estado da Bahia - FAPESB.

\section{REFERÊNCIAS}

[1] IBGE. Instituto Brasileiro de Geografia e Estatística. Pesquisa Nacional por Amostra de Domicilio (PNAD) - Segurança Alimentar e Nutricional 2004-2013. Rio de Janeiro: IBGE; 2014.

[2] Kepple A, Segal-Corrêa AM, Santos LP, Del Grossi ME. O estado da segurança alimentar e nutricional no Brasil - agendas convergentes. Brasilia: FAO-Brasil; 2015.

[3] Rasella D, Aquino R, Santos CA, Paes-Sousa R, Barreto ML. Effect of a conditional cash transfer programme on childhood mortality: a nationwide analysis of Brazilian municipalities. The Lancet. 2013;382:57-64.

[4] Food and Agriculture Organization. The State of Food Insecurity in the World. Rome: FAO; 2000.

[5] Pangaribowo EH, Gerber N, Torero M. Food and Nutrition Security Indicators: A Review. ZEF Working Paper. 2013;108.

[6] Perez-Escamilla R, Segall-Correa AM. Food insecurity measurement and indicators. Revista de Nutrição. 2008;21:15s-26s.

[7] Santos SMC, Santos LMP. Aspectos metodológicos para a avaliação de políticas públicas de Segurança Alimentar e Nutricional. In: Santos SMC, Santos LMP. Avaliação de Políticas Públicas de Segurança Alimentar e Nutricional no Estado da Bahia. 2. ed. São Paulo: Prol Editora Gráfica; 2008. p. 27-54.

[8] Kepple AW, Segall-Correa AM. Conceituando e medindo segurança alimentar e nutricional. Ciência \& Saúde Coletiva. 2011;16:187-199.

[9] Carvalho ATD, Almeida ERD, Nilson EAF, Ubarana JÁ, Fernández IM, Immink M. Métodos de análise em programas de segurança alimentar e nutricional: uma experiência no Brasil. Ciência \& Saúde Coletiva. 2013;18:309-321.

[10] Gubert MB, Benício MHD, Dos Santos LMP. Estimates of severe food insecurity in Brazilian municipalities. Cadernos de Saúde Pública. 2010;26:1595-1605.

[11] Food and Agriculture Organization. Comité de Seguridad Alimentaria Mundial. Indicadores básicos propuestos para vigilar la situación de la seguridad alimentaría. Roma: FAO; 2000.

[12] Morón C. Guía para la gestión municipal de programas de seguridad alimentaria y nutrición. Santiago: FAO, Oficina Regional para América Latina y el Caribe; 2001. 
[13] Panelli-Martins BE, Santos SMC, Assis AMO. Segurança alimentar e nutricional: desenvolvimento de indicadores e experimentação em um município da Bahia, Brasil. Rev. Nutr. 2008;21:65-81.

[14] Ribeiro JCS. Avaliação da insegurança alimentar e nutricional em um consórcio de segurança alimentar e desenvolvimento local CONSAD na Bahia [dissertação]. Salvador: Universidade Federal da Bahia; 2012.

[15] Pereira MHQ. Avaliação da Segurança Alimentar e Nutricional: Contribuições em âmbito municipal [dissertação]. Salvador: Universidade Federal da Bahia; 2014.

[16] Pimentel FC, Albuquerque PCD, Martelli PJDL, Souza WVD, Acioli RML. Caracterização do processo de trabalho das equipes de saúde bucal em municípios de Pernambuco, Brasil, segundo porte populacional: da articulação comunitária à organização do atendimento clínico. Cadernos de Saúde Pública. 2012;28.

[17] Bahia. Secretaria do Planejamento. Territórios de Identidade [nternet]. 2013 [acesso em 10 out 2013. Disponível em: http://www.seplan.ba.gov.br/territorios-de-identidade

[18] Calmon SL, Santos SMC, Pereira MHQ. Acesso aos alimentos e segurança alimentar e nutricional: avaliação da situação em municípios baianos. Demetra: Food, Nutrition and Heath. 2016;11:1351-1366.

[19] Jannuzzi PM. Indicadores para diagnóstico, monitoramento e avaliação de programas sociais no Brasil. Revista do Serviço Público. 2005;56:137-160.

[20] Santos SMC. Relatório de Pesquisa. Avaliação da Segurança Alimentar e Nutricional em Âmbito Municipal no Estado da Bahia: Aplicação, Avaliação e Reajustes de um Protocolo de Indicadores. Salvador: Escola de Nutrição/Universidade Federal da Bahia; 2010.

[21] Hackett M, Melgar-Quiñonez H, Álvarez MC. Household food insecurity associated with stunting and underweight among preschool children in Antioquia, Colombia. Revista Panamericana de Salud Pública. 2009;25:506-510.

[22] Rossi M, Ferre Z, Curuchet MR, Giménez A, Ares G. Influence of socio-demographic characteristics on different dimensions of household food insecurity in Montevideo, Uruguay. Public Health Nutrition. 2017;20(4):620-629.

[23] Sandoval L, Carpio CE. Measuring Prevalence, Profiling and Evaluating the Potential of Policy Impacts using Two Food Security Indicators in Guatemala. In: 2017 Annual Meeting, February 4-7, 2017, Mobile, Alabama. Southern Agricultural Economics Association; 2017.

[24] Food and Agriculture Organization. IFAD and WFP. 2015. The State of Food Insecurity in the World 2015. Meeting the 2015 international hunger targets: taking stock of uneven progress. Rome: FAO; 2015.
[25] De Toledo Vianna RP, Hromi-Fiedler AJ, Segall-Correa AM, Pérez-Escamilla R. Household food insecurity in small municipalities in Northeastern Brazil: a validation study. Food Security. 2012;4:295-303.

[26] De Souza Bittencourt L, Santos SMC, Jesus Pinto E, Aliaga MA, Ribeiro-Silva CR. Factors associated with food insecurity of households of public schools students in the City of Salvador, Bahia. Journal of Health, Population and Nutrition. 2013;1:471.

[27] Hoffmann R. Determinantes da insegurança alimentar no Brasil: análise dos dados da PNAD de 2004. Segur. Aliment. Nutr. 2008;15:49-61.

[28] Aliaga MA, Chaves-dos-Santos SM. Food and nutrition security public initiatives from a human and socioeconomic development perspective: Mapping experiences within the 1996 World Food Summit signatories. Social Science \& Medicine. 2014;104:74-79.

[29] Dos Santos AR, Vieira NS, Sant'Ana TT. Agricultura familiar e segurança alimentar e nutricional: análise dos resultados do Programa de Aquisição de Alimentos (PAA Doação Simultânea) nos Estados da Bahia e Minas Gerais. Cadernos Gestão Social. 2012;3:9-24.

[30] Silva V. O Papel do Programa de Aquisição de alimentos PAA - para o fortalecimento da agricultura familiar: o caso da Cooperativa da Agricultura Familiar Rural Integrada - COOPAFI - de Capanema, PR [dissertação]. Pato Branco: Universidade Tecnológica Federal do Paraná; 2013.

[31] Baiphethi MN, Jacobs PT. The contribution of subsistence farming to food security in South Africa. Agrekon. 2009;48:459482.

[32] Monego ET, Peixoto MDRG, De Morais Cordeiro M, Costa R. M. (In)segurança alimentar de comunidades quilombolas do Tocantins. Segur. Aliment. Nutr. 2010;17:37-47.

[33] Bartram J, Lewis K, Lenton R, Wright, A. Focusing on improved water and sanitation for health. The Lancet. 2005;365:810.

[34] Tingay RS, Tan CJ, Tan NCW. et al. Food insecurity and low income in an English inner city. Journal of Public Health. 2003;25:156-159.

[35] Babatunde RO, Qaim M. Impact of off-farm income on food security and nutrition in Nigeria. Food Policy. 2010;35:303-311.

[36] Cabral CS, Lopes AG, Lopes JM, Vianna RPDT. Food security, income, and the Bolsa Família program: a cohort study of municipalities in Paraíba State, Brazil, 2005-2011. Cadernos de Saúde Pública. 2014;30:393-402.

[37] Dos Santos Interlenghi G, Salles-Costa R. Inverse association between social support and household food insecurity in a 
metropolitan area of Rio de Janeiro, Brazil. Public Health Nutrition. 2015;18:2925-2933.

[38] Bezerra TA, Olinda RA, Pedraza DF. Insegurança alimentar no Brasil segundo diferentes cenários sociodemográficos. Ciência \& Saúde Coletiva. 2017;22:637-651. 